\title{
SOME PROPHYLACTIC MEASURES CAUSING INCREASED NEUROMUSCULAR ABILITY IN THE SPORTSMAN
}

\author{
M. D. AZATJAN, M.D. \\ Georgian Scientific Research Institute of Physical Culture, \\ Ronstituzi 6, Tbilisy, Georgiz, U.S.S.R.
}

it is well-known that switching over from one kind of activity to another, can develop the total productivity of work with less fatigue, and the nature of this phenomena has been understood only recently.

Many experiments have shown that the phenomena of switching over to another activity is effective only in prolonged sporting exercises; for instance, momentary back-pedalling on a bicycle while freewheeling removes early fatigue and increases work output.

A rational explanation of this phenomena is not quite clear, but experiments suggest that functions of muscles statically contracting are changed as well, leading to withdrawal of fatigue. Afterwards when switching over to active work, the motor neurones and muscle fibres that were inactive, begin to work, but the duration of the active process may vary during each cycle. For example, if one recorded the biopotential of muscles of the lower extremities: the quadriceps femoris, biceps femoris, tibialis anterior and gastrocnemius, one can see, that in the last case the correlation between the period of activation and the period of silence of each group is quite different. While the usual forward pedalling biceps femoris works through 1900 , but during back-pedalling it only works through $160^{\circ}$. In this. case when we change the direction of pedalling that is the direction of the muscle, work diminishes; that means that the muscle is in better condition for recovery.

Life of modern men becomes more and more intensive. But this intensification causes neuro-psychical excitement while physically man is relatively in the condition of immobility. Under these conditions the transfer of different actions for the same muscles has the desired effect for raising the total and special ability of the function of man. 